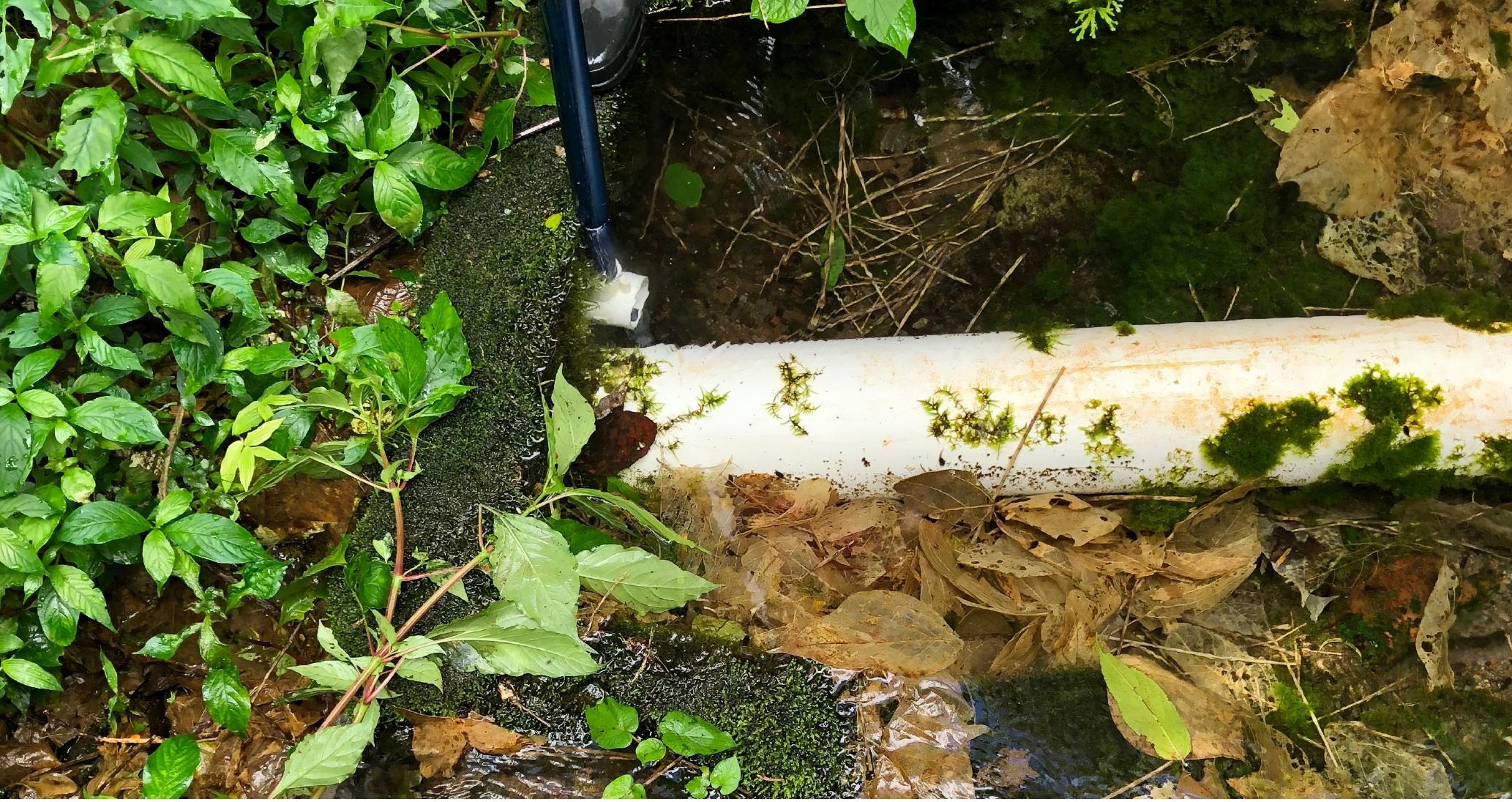

\title{
Disponibilidad del manantial "Ojo de Agua" y ahorro del uso doméstico del recurso hídrico en la localidad de Zoncuantla, Coatepec, Veracruz, México
}

S. Menchaca ${ }^{1}$ - A. Calva ${ }^{1}$ - H. Hernández ${ }^{1}$

RESUMEN: En este artículo se presentan los resultados de un estudio que se realizó en el contexto de la disponibilidad del recurso hídrico del manantial "Ojo de Agua" ubicado en el municipio de San Andrés Tlalnelhuayocan, en cuanto al atributo de cantidad y a la medición del flujo de retorno al ecosistema, también llamado caudal ecológico. También se determinaron las actividades relacionadas con el ahorro de agua para uso doméstico, que realizan los habitantes de la Congregación de Zoncuantla, Coatepec, Veracruz, los cuales se abastecen de dicho manantial. Las conclusiones permitirán tener una comprensión amplia sobre el comportamiento de los cuerpos de agua naturales y su uso.

Palabras Clave: Disponibilidad del agua, caudal ecológico, ahorro de agua, manantial, uso de agua doméstico.
ABSTRACT: This article presents the results of a study that was carried out in the context of availability of water resource of the "Ojo de Agua" spring located in the municipality of San Andrés Tlalnelhuayocan, regarding the quantity attribute and the measurement of return flow to the ecosystem, also called ecological flow. Also, the activities related to water saving for domestic use were determined, accomplished by the inhabitants of the Congregation of Zoncuantla, Coatepec, Veracruz, which is supplied by said water spring. Conclusions will allow a broad understanding of the behavior of natural water bodies and their use.

Keywords: Water availability, ecological flow, water saving, natural spring, domestic water use.

1 Observatorio del Agua para el Estado de Veracruz, OABCC (Agua, Bosques, Cuencas y Costas). Contacto: socorro. menchaca@gmail.com 


\section{Introducción}

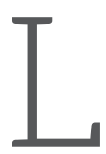
a disponibilidad del agua en el mundo es crítica y cada vez más compleja, ya que está disminuyendo en cantidad y calidad, debido a múltiples factores tanto naturales como antrópicos. En el contexto de México, entre los años 2000 y 2005, la disponibilidad anual por habitante disminuyó de 4841 a 4573 m3; respecto a lo anterior, las predicciones que establece la Comisión Nacional del Agua (CONAGUA) muestran que, debido al crecimiento poblacional, para el año 2030 la disponibilidad anual media de agua por habitante se reducirá a 3705 m3 (Plan Nacional de Desarrollo, 20072012).

En lo anterior, radica la importancia de establecer nuevos enfoques en el desarrollo de políticas, estrategias y/o acciones dirigidas a la gestión, manejo y usos de los cuerpos naturales, tales como: proteger y/o conservar las funciones de los ecosistemas y los cuerpos de agua, medir de manera permanente la disponibilidad del recurso hídrico; asegurar la cantidad y calidad el recurso para el consumo humano; mejorar la eficiencia en la administración, gestión y participación de los usuarios, y fomentar el desarrollo de actitudes, hábitos, valores y costumbres encaminadas al ahorro del agua (Menchaca, 2016).

Una de las estrategias de carácter sustentable para la conservación de cuerpos de agua como los manantiales, es la medición permanente del flujo de retorno al ecosistema, también llamado caudal ecológico. El caudal ecológico es un instrumento de gestión y manejo que permite mantener los componentes, funciones, procesos y la resiliencia de los ecosistemas acuáticos; proporcionando la conservación y preservación de especies nativas de flora y fauna, y contribuyendo a establecer el caudal mínimo necesario que debe circular en una fuente hídrica, para que se mantenga la vida acuática (Izquierdo y Madroñero, 2014).

Cabe señalar que, en México no hay una política que asegure la medición permanente de la disponibilidad del agua de los manantiales y del caudal ecológico o de retorno del recurso al medio ambiente. Lo anterior, es fundamental para la conservación del sistema ecohidrológico, cuestión que es importante ya que: permite la protección de sus funciones, expresadas en los múltiples servicios ambientales de cuencas hidrológicas y bosques, para mantener la vida de la flora y fauna del planeta; la vida y el bienestar humano depende de que se cuente con la suficiente agua para el desarrollo de las necesidades básicas; el recurso hídrico es fundamental para la producción de bienes y servicios.

Aunado a lo anterior, las instancias gubernamentales tampoco diagnostican las prácticas relacionadas con la cultura de ahorro del agua, que realizan los usuarios domésticos en las localidades y/o comunidades. Se considera que esta es una política básica para el desarrollo de una nueva cultura de cuidado del agua, en este momento histórico, en donde se considera crucial hacer conciencia y desarrollar valores, actitudes, actividades, etc., dirigidas a preservar el recurso hídrico, los cuerpos naturales, para las generaciones presentes y futuras.

Con base en lo anterior, el presente estudio se centra, por una parte, en el análisis sobre la disponibilidad del agua del manantial "Ojo de Agua", del caudal para el abastecimiento doméstico y ecológico, como el aporte de éste al arroyo Tixtla, mismo que es vital para la ecohidrología de la zona, y por la otra, en el análisis sobre las prácticas que se realizan en el contexto de ahorro del recurso hídrico del manantial, por parte de los habitantes de la Congregación de Zoncuantla, Coatepec.

\section{Material y métodos}

\subsection{Descripción de la zona de estudio}

El presente trabajo se desarrolló en el contexto de la interacción entre el manantial "Ojo de Agua" y los usos domésticos de los habitantes de la Congregación de Zoncuantla, Coatepec, Veracruz. Ambos se ubican en la cuenca alta del río La Antigua, en la que se ubica la microcuenca del río Pixquiac. Esta integra parcialmente territorios de los municipios de Perote, Las Vigas, Acajete, Coatepec y San Andrés Tlalnelhuayocan. 
El manatial "Ojo de Agua" suministra a la comunidad de Zoncuantla del recurso hídrico. Su afluente se encausó, mediante la construcción de obra hidráulica; la cual incluye: una caja de captación, conectada con una tubería de 4 pulgadas de diámetro a una caja rompedora de presión que distribuye el flujo, hacia el tanque de abastecimiento ubicado en la Congregación de Zoncuantla y, el excedente, (caudal ecológico, definición de NMX-AA-159-SCFI-2012) lo encausa al arroyo Tixtla. Con objeto de subsanar la ruptura de la caja de captación, se construyeron, como obras complementarias, dos bardas laterales de contención lateral.

La Congregación de Zoncuantla, tiene una población aproximada de 1584 habitantes (INEGI, 2010), distribuidos en cinco colonias: Mariano Escobedo, El Atorón, 6 de enero, La Pitahaya y Plan de la Cruz. La composición social es una mezcla de los distintos niveles socioeconómicos; el grado de escolaridad registrado abarca desde primaria incompleta hasta doctorado; la ocupación de los habitantes es diversa, desde personas dedicadas a oficios como construcción y servicios domésticos, hasta artistas, académicos, funcionarios públicos y profesionales de distintas áreas.

La población gestionó ante CONAGUA, hace más de veinte años, la concesión del manantial "Ojo de Agua", y hasta la fecha es la única fuente de abastecimiento para uso doméstico. La gestión y manejo de esta concesión está a cargo de la Comisión Municipal de Agua y Sa- neamiento de Coatepec.

2.2 Determinación de la disponibilidad del manantial "Ojo de Agua": uso doméstico y caudal ecológico, como aporte al arroyo Tixtla

La disponibilidad del agua del manantial, el caudal de abastecimiento y ecológico, así como su aporte al arroyo Tixtla, se determinaron mediante un balance hídrico dinámico de los aforos mensualmente de seis puntos (Figura 1). Estos se monitorearon durante el periodo comprendido de enero de 2016 a mayo de 2018, en el contexto del trabajo del Observatorio del Agua para el Estado de Veracruz, OABCC (Agua, Bosques, Cuencas y Costas) Centro de Ciencias de la Tierra, de la Universidad Veracruzana.

Para realizar el balance, se elaboró un diagrama de flujo del manantial con base en su estructura hidráulica, en el cual se establecieron los siguientes puntos de monitoreo: el punto 1 corresponde al nacimiento del manantial "Ojo de Agua", mismo que tiene un afluente alterno; los puntos 2, 3 y 4 corresponden a los tubos que encausan el agua hacia la caja rompedora de presión, la que alimenta la línea de conducción, que llega al tanque de abastecimiento de agua de la Congregación de Zoncualtla; el punto 5, es el aporte al arroyo y, por último, el punto 6 que se ubica aguas abajo del punto de mezcla del aporte del manantial y el arroyo Tixtla. (Figura 1).

Figura 1. Diagrama de flujo y puntos de monitoreo del sistema hidráulico del manantial "Ojo de Agua"

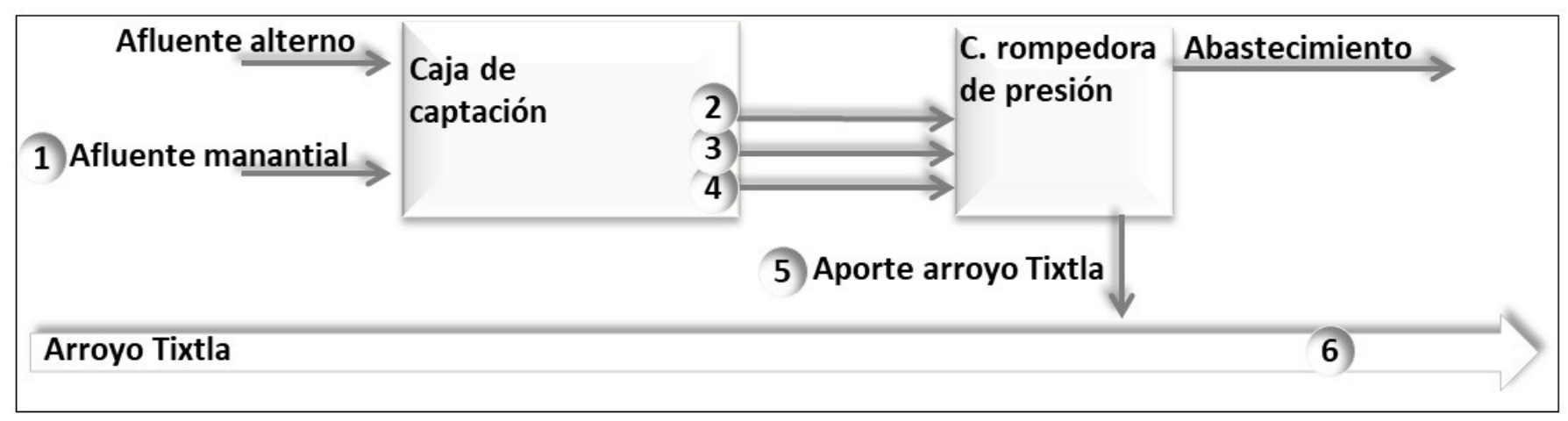

Fuente: Observatorio del Agua para el Estado de Veracruz, OABCC (Menchaca, Calva, 2017). 
Para determinar la velocidad del flujo se utilizó un flujómetro marca Global Water modelo FP201. En los puntos de monitoreo de sección circular (2, 3, 4 y 5) el área transversal del flujo se obtuvo restando el área del segmento circular sin flujo al área transversal total del tubo; mientras que, para el manantial (punto 1) y el arroyo Tixtla (punto 6), el área transversal se aproximó asumiendo la formación de un canal trapezoidal; para todos los puntos, el caudal se aproximó mediante el producto del área transversal por la velocidad de flujo. La dinámica del sistema hídrico definido se analizó mediante los gráficos de la variación mensual de los caudales en los distintos puntos del sistema, estos datos se analizaron junto con la precipitación promedio anual (datos climáticos, programa CLICOM del Sistema Meteorológico Nacional a través de la plataforma del Centro de Investigaciones Científicas y de Educación Superior de Ensenada, CICESE, periodo 2003 a 2015). Posteriormente, mediante la aplicación de la metodología propuesta por García et al. (1999), publicada en la NMX-AA-150-SCFI-2012, se calculó el caudal mínimo mensual tanto del manantial "Ojo de Agua", como del Arroyo Tixtla.

2.3 Determinación del ahorro del recurso hídrico del manantial, por parte de los habitantes de la Congregación de Zoncuanta, Coatepec.

En cuanto al ahorro de agua en la Congregación de Zoncuantla, éste se determinó con base en las siguientes categorías de análisis: el cuidado del agua, así como la revisión y mantenimiento de la instalación hidráulica en las casas, mismas que fueron medidas por distintos indicadores. Para ello, se elaboró un cuestionario para recabar información de dicha población, respecto a las prácticas que se desarrollan en relación al ahorro, el que está dirigido a evitar su desperdicio y evitar la escasez del recurso natural. El tamaño de muestra se calculó mediante la ecuación propuesta por Ochoa, (2013).

$$
\mathrm{n}=\frac{\mathrm{N} \cdot \mathrm{Z}^{2} \cdot \mathrm{p} \cdot(1-\mathrm{p})}{(\mathrm{N}-1) \cdot \mathrm{e}^{2}+\mathrm{Z}^{2} \cdot \mathrm{p} \cdot(1-\mathrm{p})}
$$

En donde: "n" representa el tamaño de muestra; "N" es el tamaño del universo (población total de la Congregación de Zoncuantla); "Z" corresponde al nivel de confianza (95\%); "e" es el margen de error (0.15) y "p" es la probabilidad de éxito o proporción esperada (0.5). El total de encuestas se distribuyó de manera proporcional entre las cinco colonias que integran la Congregación. Las encuestas se aplicaron durante los meses de noviembre y diciembre de 2016 de manera aleatoria, como criterio de aplicación se usó: "personas que accedieran a participar en la encuesta, sin distinción de edad, sexo, nivel socioeconómico o tiempo de residencia en la comunidad". Con la información obtenida se desarrolló una base de datos en relación a las categorías de análisis y los indicadores con las que fueron evaluadas; posteriormente se realizó un análisis porcentual de las respuestas de los entrevistados, en el contexto del ahorro del recurso que se realiza en la Congregación de Zoncuantla, Coatepec, Veracruz.

\section{Resultados y análisis}

\subsection{Disponibilidad del agua y caudal ecológico}

El balance mostró una variación mensual significativa del caudal del manantial "Ojo de Agua", que van desde 4.5 hasta $27.3 \mathrm{~L} \mathrm{~s}^{-1}$; con un promedio de $10.8 \mathrm{~L} \mathrm{~s}^{-1}$. Su aporte representa, en promedio, el $30 \%$ del caudal de entrada a la caja rompedora de presión; el resto (70 \%) proviene de un afluente alterno, el cual, puede estar conformado por agua de escorrentía y/o filtración. Al comparar las variaciones mensuales tanto del manantial "Ojo de Agua", como del afluente alterno, con el histórico de la precipitación pluvial, no se observó una correlación entre éstos ( $\mathrm{p} \geq 0.1)$ (Figura 2).

Sin embargo, el caudal de ambos afluentes, así como su variación, aumentaron significativamente durante los meses de lluvia (junio septiembre). La dinámica del manantial mostró que durante los meses de enero a julio el caudal promedio fue de 8 L s-1; posteriormente, de agosto a octubre tuvo un incremento de $10 \mathrm{~L}$ 
$\mathrm{S}^{-1}$ respectivamente, para mantener un caudal promedio de $16 \mathrm{~L} \mathrm{~s}^{-1}$ durante los últimos tres meses del año; probablemente por el efecto tardío de la temporada de lluvia. Un comportamiento similar se observó para el afluente alterno (Figura 3). 3.2 Caudal de abastecimiento a la comunidad, caudal ecológico del manantial "Ojo de Agua" y del arroyo Tixtla

Teniendo como referencia que el aporte del manantial "Ojo de Agua" junto con el afluente alterno a la caja rompedora de presión tiene un flujo que va desde 21.8 hasta $52.4 \mathrm{~L} \mathrm{~s}^{-1}$, y que una parte de ese flujo alimenta la línea de conducción que llega al tanque de abastecimiento de agua de la Congregación de Zoncuantla, y el resto corresponde al flujo de retorno o caudal ecológico del manantial, el cual aporta recurso hídrico al arroyo Tixtla, se tiene que el balance hídrico del caudal de abastecimiento a la comunidad varió desde 21 hasta $41.1 \mathrm{~L} \mathrm{~s}^{-1}$ con un promedio $27.5 \mathrm{~L} \mathrm{~s}^{-1}$, lo cual representa el $82 \%$ del total de agua que entra en la caja de captación. Por otro lado, el balance hídrico del aporte al arrollo Tixtla varió desde 0 hasta $12.8 \mathrm{~L} \mathrm{~s}^{-1} \mathrm{con}$ un promedio de $5.7 \mathrm{~L} \mathrm{~s}^{-1}$, lo cual representa únicamente el $17 \%$ del total de agua que entra en la caja de captación (Figura 4).

Figura 2. Relación de la precipitación pluvial histórica de Coatepec, Veracruz con el caudal del manantial "Ojo de Agua" y el afluente alterno (Promedios del periodo 2016-2018).

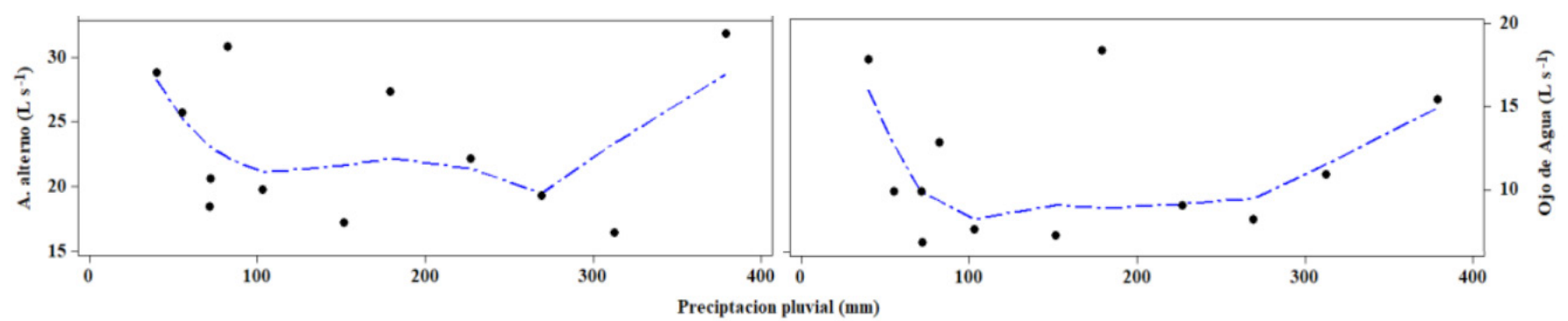

Figura 3. Comparación del comportamiento anual del manantial y el afluente alterno con la precipitación pluvial promedio (periodo 2016-2018).

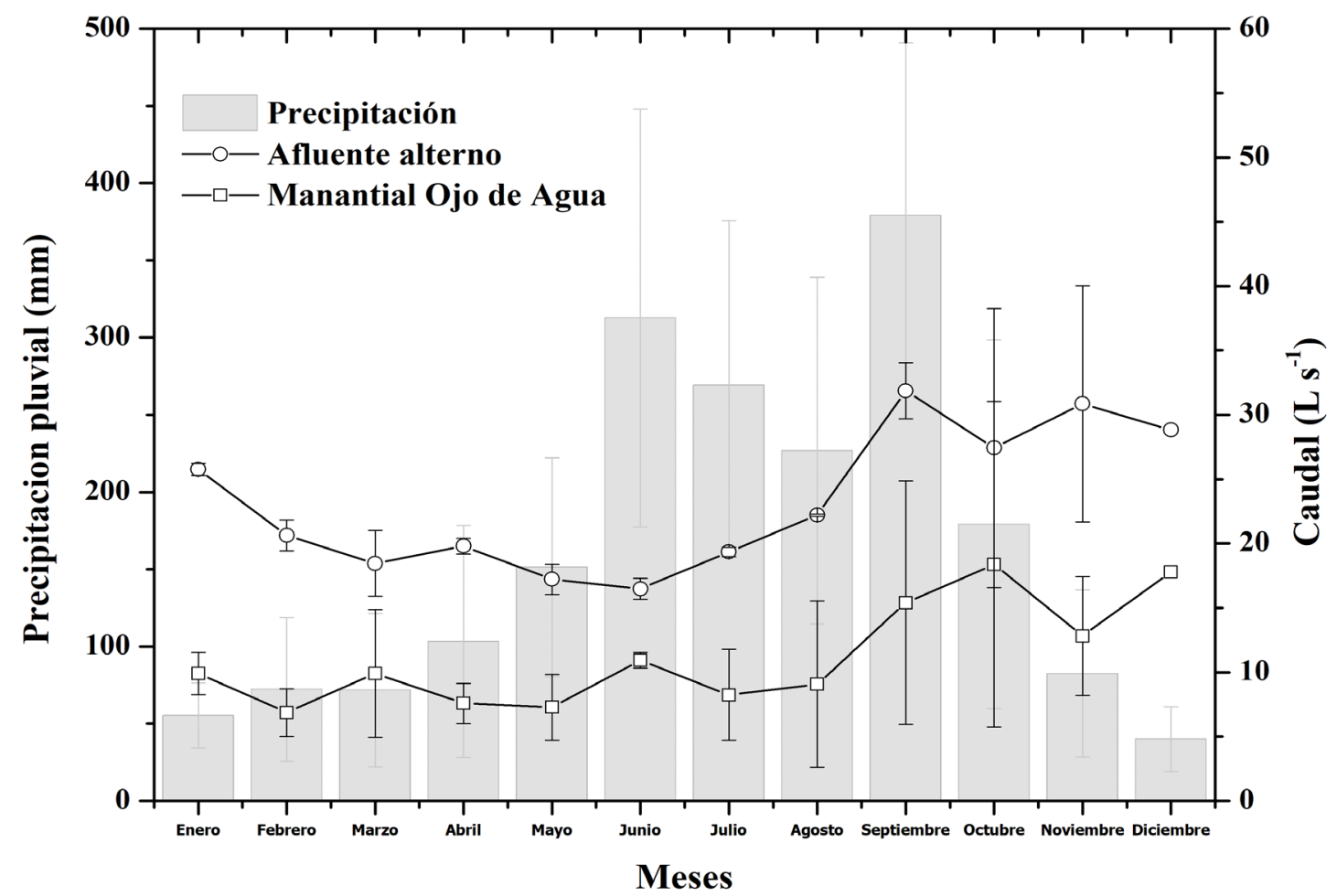


Figura 4. Análisis del régimen anual de caudales para la entrada a la caja rompedora de presión, el de abastecimiento a la comunidad y el caudal ecológico (promedios del periodo 2016-2018).

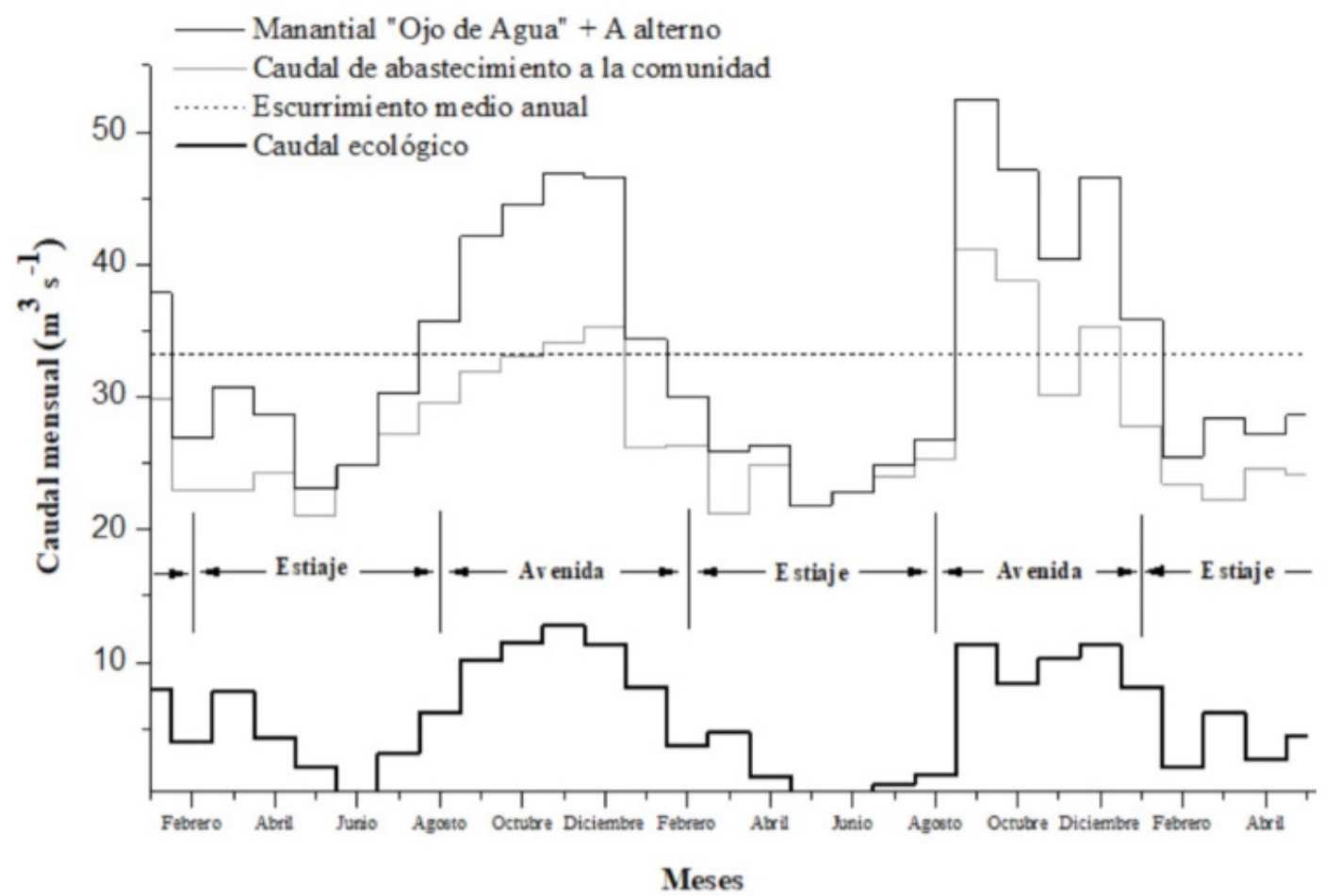

Se observó que; en los meses de mayo de 2017 y junio de ambos años, el caudal de la caja rompedora de presión es igual al caudal de abastecimiento a la comunidad; es decir, no hay un aporte al arroyo Tixtla, por parte del manantial y ello pone en riesgo las funciones y procesos del ecosistema que proporcionan bienes y servicios a la sociedad (Figura 5). El manantial "Ojo de Agua" pertenece a la subcuenca del río Pixquiac, la cual está dentro de la cuenca alta del río La Antigua (RH-28). La cuenca, está considerada para cumplimiento de objetivo ambiental tipo "A" (NMX-AA-150SCFI-2012). Esta denotación implica: la existencia de especies endémicas y/o de relevancia internacional, nula o mínima alteración ecohidrológica, es decir, que aún conserva sus servicios ecosistémicos (alta integridad ecológica). El análisis de la dinámica del caudal del manantial y el afluente alterno en conjunto, mostró que el caudal ecológico, que actualmente tiene, es significativamente menor al necesario ( $F$ igura 5). La norma sugiere que el caudal ecológico no debe ser menor que el caudal base; sin embargo, para el caso del manantial no se cumple (Figura 5). Esto se debe a que la mayor parte del flujo del manantial se envía como recurso a la comunidad; ocasionando que la presión de uso sobre el manantial sea muy alta (82 \%). Es importante mencionar que la cuenca a la que pertenece este cuerpo de agua debe de tener una presión de uso media (entre 11 y 40 \%).

La importancia de mantener el caudal ecológico del manantial radica en el efecto que tienen sobre el arroyo Tixtla. Sin este aporte, el arroyo no alcanza su caudal ecológico durante los meses de febrero, marzo, abril, mayo, julio y agosto. Por otro lado, con el aporte del manantial, mantiene el caudal ecológico durante todo el año, y en los meses de enero, septiembre, octubre, noviembre y diciembre tiene un flujo mayor al caudal ecológico (Figura 6).

3.3 Ahorro de agua en la Congregación de Zoncuantla

A continuación se presenta la información relativa al ahorro de agua del manantial de interés, en relación a las categorías de análisis: el cuidado del agua, así como la revisión y mantenimiento de la instalación hidráulica en las casas, y respectivos indicadores.

A. Respecto al ahorro en relación con el cui- 
Figura 5. Análisis del régimen anual de caudales para el manantial "Ojo de Agua" y el afluente alterno (promedios del periodo 2016-2018).

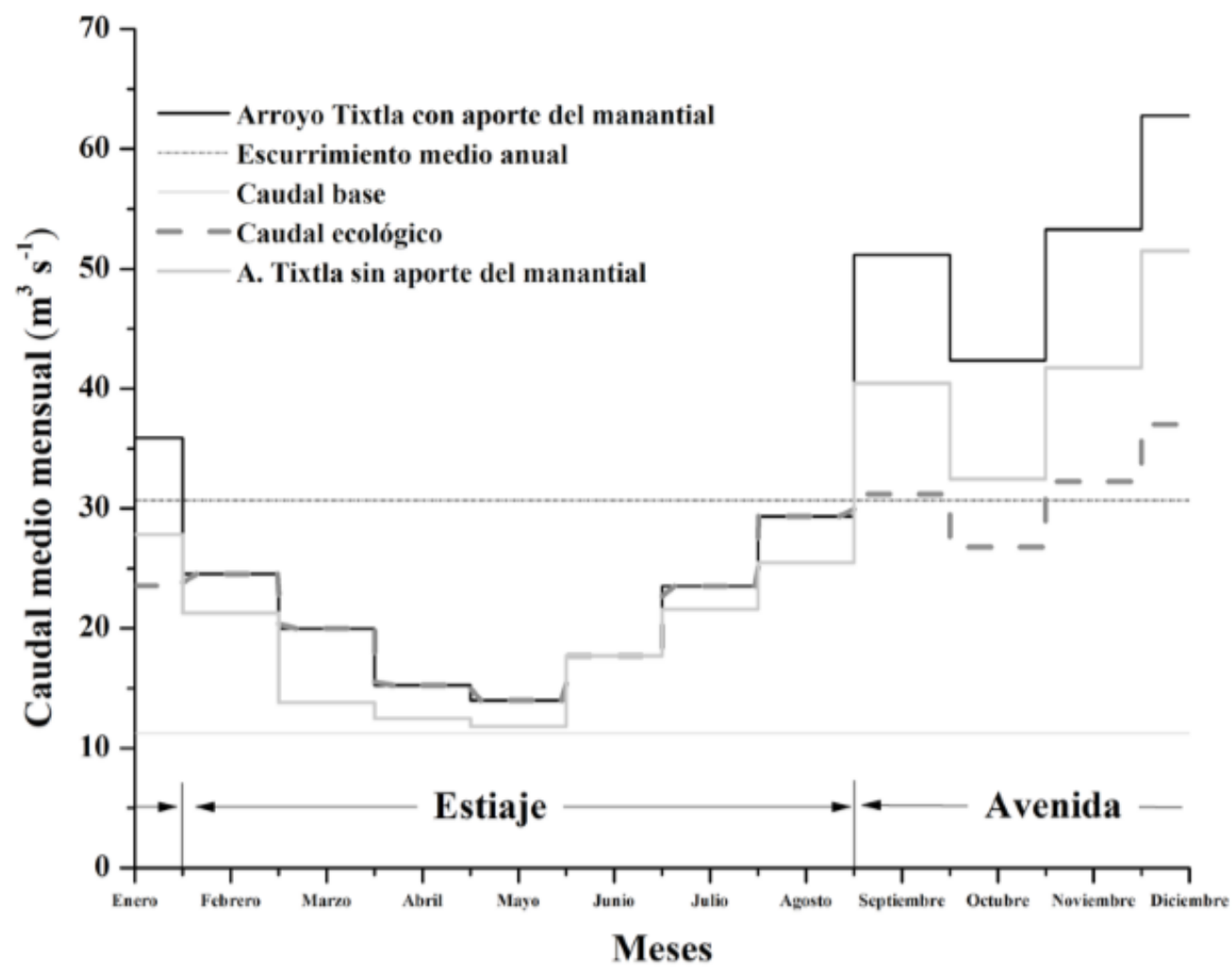

Figura 6. Análisis del régimen del caudal ecológico para el arroyo Tixtla, con y sin el aporte del manantial "Ojo de Agua” (promedios del periodo 2016-2018).

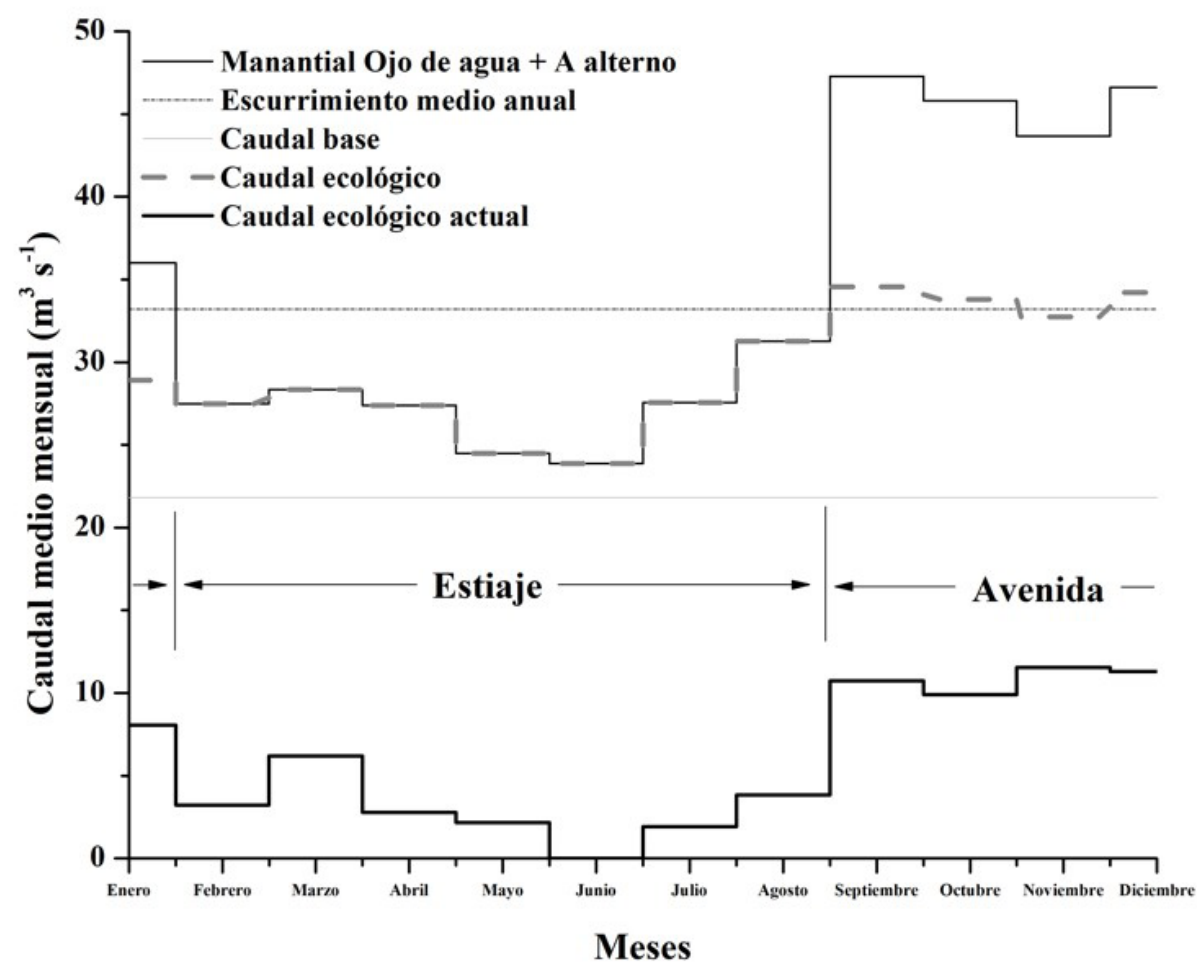


Figura 7. Cuidado del agua.

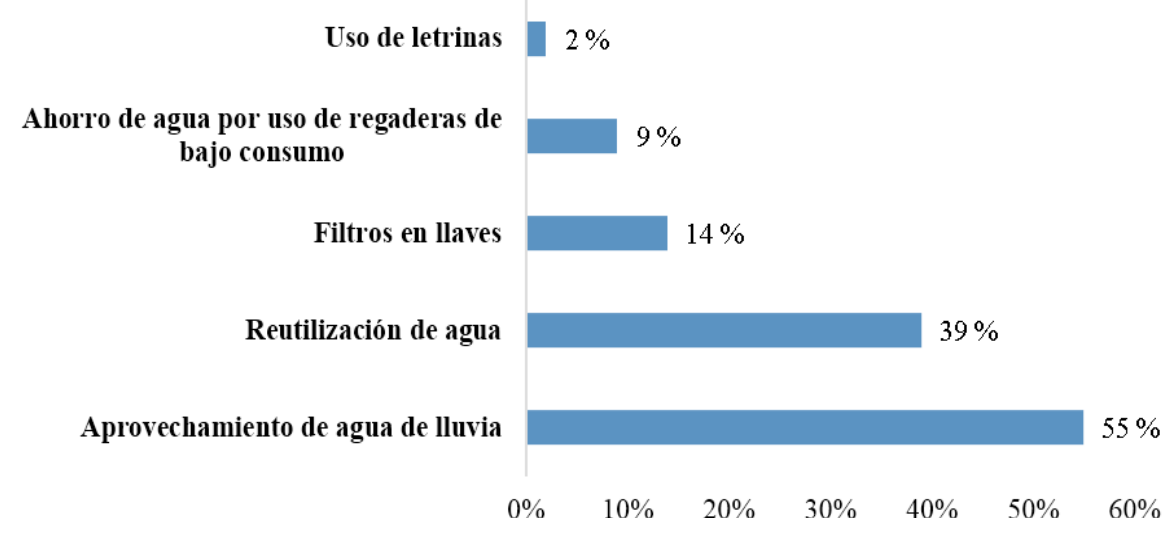

Fuente: Observatorio del Agua para el Estado de Veracruz, OABCC, Menchaca, Calva, 2018

dado del agua, se midieron las siguientes acciones: aprovechamiento de agua de lluvia; reutilización de agua; uso de filtros en llaves; ahorro de agua por el uso de regaderas de bajo consumo; y, por último, uso de letrinas.

Del total de personas entrevistadas, el $55 \%$ aprovecha el agua de lluvia para distintas actividades de uso doméstico; el $39 \%$ reutiliza el agua, lo cual, consiste en el uso de aguas grises para distintas actividades domésticas; respecto al uso de filtros en llaves, se encontró que solamente el $14 \%$ hace uso de éstos; en cuanto al ahorro de agua por el uso de regaderas de bajo consumo, tan solo el 9 \% manifestó utilizarlas; y por último, del total de entrevistados, únicamente el $2 \%$ hace uso de letrinas (Figura 7).

A continuación, se presenta el análisis de cada acción:

Aprovechamiento de agua de lluvia: la principal actividad en la cual se aprovecha el agua de lluvia es en la limpieza de la casa. También, se utiliza el agua de lluvia en el lavado del patio y/o banqueta, al igual que en el riego de plantas y/o jardín, así como el lavado de auto. Cabe mencionar que, el $55 \%$ de las personas entrevistadas, manifiestan que prefieren captar el agua de lluvia para utilizarla en dichas actividades en lugar de hacer uso de la manguera. Al respecto, Aracena (2011), menciona que el uso de manguera tiene un consumo de 20 a 30 litros por minuto, lo cual, corresponde al ahorro aproximado que se generaría al aprovechar el agua de lluvia.

Reutilización de agua: el 39 \% de las perso- nas reutilizan el agua en distintas actividades domésticas, por ejemplo: el agua que se descarga de la lavadora se utiliza en la limpieza de la casa; el agua que se ocupa para lavar frutas y verduras se usa para regar las plantas y/o jardín; en la regadera antes de bañarse, se coloca una cubeta o recipiente para no desperdiciar agua mientras llega a la temperatura deseada, para después reutilizarla, por ejemplo, en la descarga del W.C., en el riego de plantas y/o jardín, en el lavado de auto, entre otras actividades de carácter doméstico.

Filtros en llaves: se encontró que, esta acción se lleva a cabo en las llaves de los baños, en la llave del lavaplatos y/o en la manguera, ya que permite regular el flujo del agua evitando que se desperdicie. Los filtros se instalan en la salida de las llaves y con ello se consigue un ahorro aproximado del $70 \%$ de agua, además, se ahorra tiempo y por ende, se gasta menos agua (Manco, Guerrero y Ocampo, 2012). Cabe señalar que, sólo el 14 \% de los entrevistados manifestaron utilizarlos. Esto podría deberse a que en la Congregación de Zoncuantla, no se tiene suficiente información acerca de los beneficios que tiene el uso de filtros en llaves.

Ahorro de agua por el uso de regaderas de bajo consumo: se señala que, el uso de estas regaderas reduce el consumo de agua hasta un 50 \% en comparación a las regaderas convencionales, las cuales gastan de 70 a 100 litros cada 10 minutos (Vassallo, 2009). Sin embargo, se encontró que no se usan comúnmente en la Congregación de Zoncuantla, ya que, única- 


\section{Figura 8. Revisión y mantenimiento de la instalación hidráulica. Fuente: Observatorio del Agua para} el Estado de Veracruz, OABCC, Menchaca, Calva, 2018.

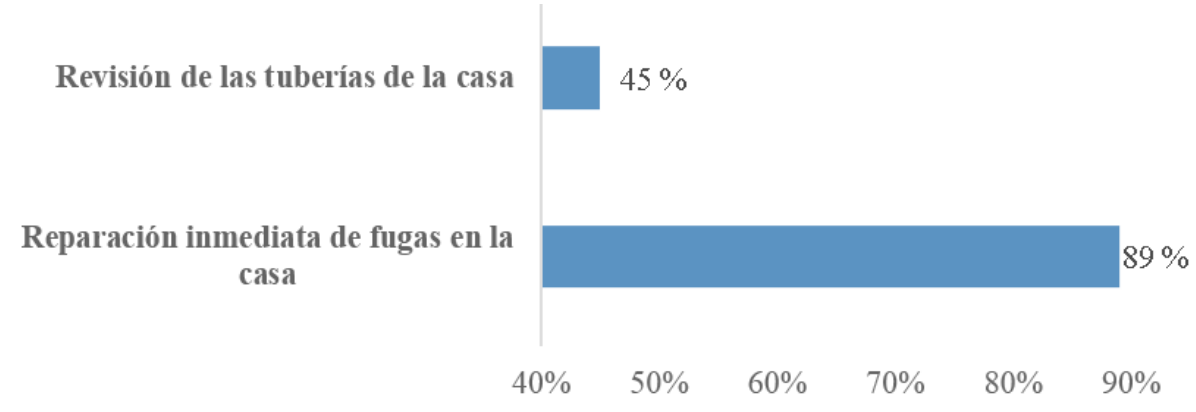

Fuente: Observatorio del Agua para el Estado de Veracruz, OABCC, Menchaca, Calva, 2018

mente el $9 \%$ manifestó utilizarlas. Esto puede deberse, como se mencionó anteriormente, a la falta de información acerca del beneficio que tienen para el ahorro del recurso hídrico.

Uso de letrinas: de todas las acciones descritas anteriormente, el uso de letrinas es la única acción en donde no se requiere agua, por lo que se considera la más importante, pues además, las excretas y/o material orgánico pueden utilizarse como abono para jardines, huertos, etc. Al respecto, Aracena (2011) señala que, un W.C. convencional consume de 18 a 20 litros de agua al tirar de la cadena, así como, un W.C. de bajo consumo tiene un gasto aproximado de 6 a 10 litros de agua. Lo anterior significa que, al usar letrinas en una familia promedio de 4 habitantes, se pueden ahorrar de 200 a 400 litros de agua diarios. Sin embargo, los resultados muestran que, solamente el $2 \%$ de los habitantes entrevistados realiza esta acción. Algunas personas relacionan el uso de letrinas o baños secos con una mala higiene, lo cual, es debido a la falta de información al respecto.

B. En cuanto al ahorro del agua relacionado con la revisión y el mantenimiento de la instalación hidráulica en las casas, se midieron las siguientes acciones: reparación inmediata de fugas en la casa y revisión periódica de las tuberías. Los resultados muestran que, del total de personas entrevistadas, el $89 \%$ repara inmediatamente las fugas en su casa; y el $45 \%$ revisa semanalmente $\mathrm{y} / \mathrm{o}$ mensualmente las tuberías de su casa (Figura 8).

A continuación, se presenta el comportamiento de cada acción:
Reparación inmediata de fugas en la casa: al respecto de esta acción, el 89 \% de las personas entrevistadas reparan inmediatamente las fugas en su casa. En términos generales, uno de los lugares que más fugas presenta es el W.C., gastando aproximadamente 250 litros de agua al mes (Aracena, 2011). Por otro lado, las llaves de lavabo, lavaplatos o regaderas también pueden presentar fugas debido al desgaste del empaque, lo que provoca que estén goteando constantemente (Arreguín, 1991). Cabe señalar que, una llave que gotea tiene un gasto aproximado de 30 litros de agua al día, es decir, más de 10,000 litros al año (Aracena, 2011).

Revisión de las tuberías de la casa: esta actividad se considera muy importante y necesaria, ya que con ello, se pueden detener las fugas, al igual que reemplazar piezas viejas de la instalación hidráulica para una mayor eficiencia y ahorro. Sin embargo, solamente el 45 \% de las personas manifiestan revisar las tuberías de su casa con esta frecuencia. Cabe señalar que, no se tienen datos en cuanto al número de litros de ahorro de agua generado por la revisión periódica de las tuberías, ya que este puede ser muy variable.

\section{Conclusiones}

Se considera indispensable el abastecimiento de agua a los habitantes de la Congregación de Zoncuantla, para el uso doméstico del manantial "Ojo de Agua", ya que la seguridad hídrica debe ser una prioridad para las políticas y es- 
trategias gubernamentales. Sin embargo, también se considera que, igual importancia tiene el cuidado de los cuerpos de agua y la ecohirdología de la región, cuestión que no está siendo atendida, debido a que los flujos del caudal ecológico no son los adecuados, y de mantenerse la dinámica, existe una alta probabilidad de que se pierda el aporte del manantial "Ojo de Agua" al arroyo Tixtla, lo que pondría en riesgo la integridad ecológica del arroyo, ocasionando afectaciones a los servicios ecosistémicos y a la disponibilidad del recurso natural.

Si bien se muestra en la investigación, que los habitantes desarrollan distintas actividades para ahorrar el agua, lo que puede relacionarse con una conciencia sobre la importancia del uso racional del recurso hídrico, es fundamental que se amplíen las categorías para diagnosticar y/o evaluar, la cultura del agua en cuanto al uso racional respecto a los distintos usos del recurso en el ámbito doméstico, entre otros aspectos, cuestión que el Observatorio del Agua para el Estado de Veracruz, OABCC (Agua, Bosques, Cuencas y Costas), actualmente está estudiando, para tener un panorama completo respecto a la problemática relativa al uso doméstico del recurso y los cuerpos de agua naturales.

Se recomienda implementar campañas de cultura del agua en la Congregación de Zoncuantla, transfiriendo a la población la información más relevante respecto a las condiciones en las que se encuentra el manantial y la disponibilidad del agua, ya que es importante que los habitantes tengan conocimiento acerca de la problemática ambiental actual, así como, establezcan acciones en torno al ahorro del agua. Una correcta propuesta e implementación de estrategias permitiría, la disminución de la presión de uso sobre el manantial, coadyuvando a asegurar la continuidad del recurso y mantener los componentes, funciones y procesos de los ecosistemas acuáticos de la zona de estudio.

\section{Referencias}

Aracena, G. (2011). Manual del Consumo Responsable para el Hogar (Superintendencia de Servicios Sanitarios) (p. 46). Chile.

Aranda, E. (2013). Manantial "Ojo de Agua". La historia del abasto de agua potable para la comunidad de Zoncuantla, Coatepec, Ver,. Presentado en Asociación de Vecinos del Pixquiac-Zoncuantla, A.C

Arreguín, F. (1991). Uso eficiente del agua. México: Instituto Mexicano de Tecnología del Agua, 14

CLICOM, SMN, CICESE. Datos climáticos diarios http:// clicom-mex.cicese.mx. Recuperados 19/07/18,16:00.

García, E.; González, R.; Martínez, P.; Athala, J.; y Paz, G. (1999): Guía de Aplicación de los Métodos de Cálculo de Caudales de Reserva Ecológicos en México. Comisión Nacional del Agua, Instituto Mexicano de Tecnología del Agua. México. 190 p.

INEGI. (2010). Censo de Población y Vivienda 2010. Recuperado en junio de 2018, de Censos y Conteos de Población y Vivienda: http://www3.inegi.org.mx/sistemas/iter/default.aspx?ev=5

Izquierdo, M., y Madroñero, S. (2013). Régimen de caudal ecológico, herramienta de gestión para conservar la biota acuática. Ciencia e Ingeniería Neogranadina, 23(2), 77-94.

Manco, D., Guerrero, J., y Ocampo, A. M. (2012). Eficiencia en el consumo de agua de uso residencial, Vol. 11, No. 21. Revista Ingenierías Universidad de Medellín. pp. 23-38.

Menchaca, S. (2016). Cultura del agua para la gobernanza en la gestión integral de los recursos hídricos. México: Universidad Veracruzana. 112 p.

Menchaca, S., Calva, A. (2017). Observatorio del Agua para el Estado de Veracruz, OABCC (Agua, Bosques, Cuencas y Costas) del Centro de Ciencias de la Tierra, Universidad Veracruzana.

Ochoa, C. (2013). ¿Qué tamaño de muestra necesito? Recuperado en mayo de 2018, de Netquest: http://www. netquest.com/blog/es/que-tamano-de-muestra-necesito/

Secretaría de Economía. (2012). Que establece el procedimiento para la determinación del caudal ecológico en cuencas hidrológicas. NMX-AA-159-SCFI-2012

Plan Nacional de Desarrollo. (2007, 2012). Gobierno de los Estados Unidos Mexicanos, Presidencia de la República.

Vassallo, T. (2009). Consumo de agua en el interior de la vivienda. TAE. 